\title{
Minmax via Replicator Dynamics
}

\author{
Josef Hofbauer ${ }^{1}$
}

Published online: 16 March 2018

(C) The Author(s) 2018

\begin{abstract}
I present a short proof of the minmax theorem using the replicator dynamics.
\end{abstract}
Keywords Minmax theorem $\cdot$ Zero-sum game $\cdot$ Replicator dynamics

\section{Introduction}

The minmax theorem of von Neumann [10] says that

$$
\max _{x \in X} \min _{y \in Y} U(x, y)=\min _{y \in Y} \max _{x \in X} U(x, y)
$$

where $X, Y$ are the unit simplices in $\mathbb{R}^{n}, \mathbb{R}^{m}$ and $U: X \times Y \rightarrow \mathbb{R}$ is a continuous function, quasi-concave in $x$ and quasi-convex in $y$. The proof was by induction on the number of variables, see also [7]. An important special case is where $U$ is a bilinear function $U(x, y)=$ $x \cdot A y$, with $A$ an $n \times m$ matrix.

The idea to use dynamics for proving the minmax theorem (and computing the equilibria) goes back to Brown [1,2]: for symmetric zero-sum games, i.e., $A=-A^{\top}$, he proved together with von Neumann [2] that the solutions of a certain differential equation converge to the set of equilibria. In [1], he showed that the (continuous time) fictitious play process approaches the set of equilibria in any finite zero-sum game, which implies the minmax theorem for any $n \times m$ matrix $A$. Brown's fictitious play process [1] is now often framed as best response dynamics and can be used to prove the minmax theorem for more general payoff functions $U$, which are continuous and concave/convex, see [6]. For the original version [10] for continuous quasi-concave/quasi-convex functions, a dynamic proof is still missing. Another proof based on differential inclusions can be found in [8].

In the present note, I give a short proof of the minmax theorem in the matrix case, based on the replicator dynamics.

Josef Hofbauer

josef.hofbauer@univie.ac.at

1 Department of Mathematics, University of Vienna, Oskar-Morgenstern-Platz 1, 1090 Vienna, Austria 


\section{Replicator Dynamics}

The replicator dynamics [5] for an $n \times m$ bimatrix game $(A, B)$ is given by

$$
\begin{aligned}
& \dot{x}_{i}=x_{i}\left(e^{i} \cdot A y-x \cdot A y\right) \quad i=1, \ldots, n \\
& \dot{y}_{j}=y_{j}\left(e^{j} \cdot B x-y \cdot B x\right) \quad j=1, \ldots, m
\end{aligned}
$$

Here $x_{i}$ denotes the frequency of strategy $i$ of player 1 , hence $x=\left(x_{1}, \ldots, x_{n}\right)$ is in the probability simplex $\Delta_{n}=\left\{x \in[0,1]^{n}: \sum x_{i}=1\right\}, y_{j}$ is the frequency of strategy $j$ of player $2, y=\left(y_{1}, \ldots, y_{m}\right) \in \Delta_{m}$, and $e^{i}$ denotes the $i$ th unit vector.

Besides its original derivation from evolution and natural selection, there are at least two economic motivations based on imitation and on reinforcement learning.

For a zero-sum game $B=-A^{\top}$, in the interior of $\Delta_{n} \times \Delta_{m}$ we obtain

$$
\begin{aligned}
\dot{x}_{i} / x_{i} & =e^{i} \cdot A y-x \cdot A y \quad i=1, \ldots, n \\
\dot{y}_{j} / y_{j} & =-x \cdot A e^{j}+x \cdot A y \quad j=1, \ldots, m
\end{aligned}
$$

Now add these equations

$$
\frac{\dot{x}_{i}}{x_{i}}+\frac{\dot{y}_{j}}{y_{j}}=e^{i} \cdot A y-x \cdot A e^{j} \quad \forall i, j
$$

and integrate

$$
\frac{\log x_{i}(T)-\log x_{i}(0)+\log y_{j}(T)-\log y_{j}(0)}{T}=e^{i} \cdot A \bar{y}(T)-\bar{x}(T) \cdot A e^{j}
$$

where

$$
\bar{x}(T)=\frac{1}{T} \int_{0}^{T} x(t) \mathrm{d} t, \quad \bar{y}(T)=\frac{1}{T} \int_{0}^{T} y(t) \mathrm{d} t
$$

denote time averages of the solutions of $(1,2)$. Now consider limit points, i.e., choose a sequence $T_{k} \rightarrow \infty$ s.t. $\bar{x}\left(T_{k}\right) \rightarrow \bar{x}, \bar{y}\left(T_{k}\right) \rightarrow \bar{y}$. Since $\log x_{i}(T) \leq 0$, we obtain $0 \geq$ $e^{i} \cdot A \bar{y}-\bar{x} \cdot A e^{j} \quad \forall i, j$ or

$$
e^{i} \cdot A \bar{y} \leq \bar{x} \cdot A e^{j} \quad \forall i, j .
$$

Multiplying by $x_{i}$ and $y_{j}$ and summing over $i$ and $j$, we obtain

$$
\max _{x \in \Delta_{n}} x \cdot A \bar{y} \leq \min _{y \in \Delta_{m}} \bar{x} \cdot A y
$$

and

$$
\min _{y} \max _{x} x \cdot A y \leq \max _{x} x \cdot A \bar{y} \leq \min _{y} \bar{x} \cdot A y \leq \max _{x} \min _{y} x \cdot A y,
$$

and together with the obvious inequality, we obtain

$$
\min _{y \in \Delta_{m}} \max _{x \in \Delta_{n}} x \cdot A y=\max _{x \in \Delta_{n}} \min _{y \in \Delta_{m}} x \cdot A y .
$$

Additionally, (3) or (4) also imply

$$
x \cdot A \bar{y} \leq \bar{x} \cdot A \bar{y} \leq \bar{x} \cdot A y \quad \forall x, y
$$

so $(\bar{x}, \bar{y})$ is a pair of optimal strategies for the zero-sum game. (In particular, this shows the existence of equilibria.) 
Furthermore, if we integrate $(1,2)$ directly, then we obtain $0 \geq e^{i} \cdot A \bar{y}-\bar{a}$ and $0 \geq$ $-\bar{x} \cdot A e^{j}+\bar{a}$, with $\bar{a}=\lim _{T \rightarrow \infty} \frac{1}{T} \int_{0}^{T} x(t) A y(t) \mathrm{d} t$, and hence

$$
e^{i} \cdot A \bar{y} \leq \bar{a} \leq \bar{x} \cdot A e^{j} \quad \forall i, j .
$$

Comparing with (6), we get $\bar{a}=\bar{x} \cdot A \bar{y}$.

Summarizing, besides minmax theorem (5), we have shown:

Theorem Every limit point $(\bar{x}, \bar{y})$ of the time averages $(\bar{x}(T), \bar{y}(T))$ of positive solutions $(x(t), y(t))$ of the replicator dynamics is a pair of optimal strategies of the zero-sum game. And the time averages of the payoffs

$$
\frac{1}{T} \int_{0}^{T} \sum_{i, j} a_{i j} x_{i}(t) y_{j}(t) d t
$$

converge to the value $\bar{x} \cdot A \bar{y}$ of the game, as $T \rightarrow \infty$.

\section{Remarks}

1. If $\log x_{i}(T)$ and $\log y_{j}(T)$ are bounded functions of $T$ (i.e., the solution stays at a positive distance from the boundary of $\Delta_{n}$ and $\Delta_{m}$ ), then we have equality in (3) for all $i, j$, and the existence of a fully mixed equilibrium follows. The converse holds as well, see [3,5,9]: If $(p, q)>0$ is an equilibrium of the zero-sum game, then the relative entropy

$$
H(x, y)=-\sum_{i} p_{i} \log \frac{x_{i}}{p_{i}}-\sum_{j} q_{j} \log \frac{y_{j}}{q_{j}} \geq 0
$$

or Kullback-Leibler divergence is a constant of motion for $(1,2): \dot{H}=0$. The replicator dynamics is even a Hamiltonian system w.r.t. a suitable symplectic or Poisson structure [3], and hence, on each level set of $H$, by Poincaré's recurrence theorem, almost every solution is recurrent. The behavior of the solutions might be chaotic, but by the above theorem, their time averages approach the set of equilibria.

2. For nonzero-sum games, a similar argument shows that the time averages

$$
\frac{1}{T} \int_{0}^{T} x_{i}(t) y_{j}(t) \mathrm{d} t
$$

(i.e., how often does player 1 use strategy $i$ against strategy $j$ of player 2 in a given period) converge (as $T \rightarrow \infty$ ) to the set of exact coarse correlated equilibria, see [4]. This holds also for $N$ player normal form games.

Acknowledgements Open access funding provided by University of Vienna.

Open Access This article is distributed under the terms of the Creative Commons Attribution 4.0 International License (http://creativecommons.org/licenses/by/4.0/), which permits unrestricted use, distribution, and reproduction in any medium, provided you give appropriate credit to the original author(s) and the source, provide a link to the Creative Commons license, and indicate if changes were made.

\section{References}

1. Brown GW (1951) Iterative solution of games by fictitious play. In: Koopmans TC (ed) Activity analysis of production and allocation. Wiley, New York, pp 374-376 
2. Brown GW, von Neumann J (1950) Solutions of games by differential equations. Ann. Math. Stud. 24:73-79

3. Hofbauer J (1996) Evolutionary dynamics for bimatrix games: A Hamiltonian system? J. Math. Biol. 34:675-688

4. Hofbauer J (2004) Time averages of the replicator dynamics and correlated equilibria. (Preprint)

5. Hofbauer J, Sigmund K (1998) Evolutionary games and population dynamics. Cambridge University Press, Cambridge

6. Hofbauer J, Sorin S (2006) Best response dynamics for continuous zero-sum games. Discrete Continuous Dyn Syst B 6:215-224

7. Kjeldsen TH (2001) John von Neumann's conception of the minimax theorem: a journey through different mathematical contexts. Arch Hist Exact Sci 56:39-68

8. Lehrer E, Sorin S (2007) Minmax via differential inclusion. J Convex Anal 14:271-274

9. Schuster P, Sigmund K, Hofbauer J, Wolff R (1981) Selfregulation of behaviour in animal societies. Part 2: games between two populations without selfinteraction. Biol Cybern 40:9-15

10. von Neumann J (1928) Zur Theorie der Gesellschaftsspiele. Math Annalen 100:295-320 